\title{
Investigating space-weathering on the moon using APT
}

Jennika Greer ${ }^{1}$, Surya Rout ${ }^{2}$, Dieter Isheim ${ }^{3}$, David Seidman ${ }^{3}$, Rainer Wieler ${ }^{4}$ and Philipp Heck ${ }^{5}$

${ }^{1}$ Department of the Geophysical Sciences, University of Chicago, Chicago, Illinois, 60637 USA, United States, ${ }^{2}$ Institute of Materials Physics, Helmholtz-Zentrum Geesthacht, United States, ${ }^{3}$ Northwestern University Center for Atom Probe Tomography (NUCAPT), Northwestern University, Evanston, Illinois 60208, USA, United States, ${ }^{4}$ Institute of Geochemistry and Petrology, ETH Zürich, Zürich, Switzerland, United States, ${ }^{5}$ Robert A. Pritzker Center for Meteoritics and Polar Studies, Field Museum of Natural History, Chicago, Illinois, 60605 USA, United States

The surfaces of airless bodies like the Moon are subject to space weathering, the alteration of the upper surface of materials due to the simultaneous effects of irradiation by cosmic and solar rays, electromagnetic radiation, and impacts from micrometeorites. These interactions cause physical and chemical alteration of the surface, impact vaporization, and ion sputtering and implantation, which lead to gardening and change in optical properties of the surface materials (eg., [1-4]). The total depth of this alteration can be up to $200 \mathrm{~nm}$ (or more with the presence of melt splashes and agglutinates). Atom probe tomography (APT) has the appropriate 3-D spatial resolution and analytical sensitivity to investigate such features. In APT, atoms are field evaporated from the surface of a sample and detected by a positionsensitive time-of-flight mass spectrometer. This way, both the local compositions and spatial distributions can be determined in three dimensions [5]. We have demonstrated that APT can be successfully used to characterize the composition and texture of space weathering products in ilmenite from Apollo 17 sample 71501 at near-atomic resolution [6].

Two of the studied nanotips sampled the top surface of the space-weathered grain, while another sampled the deeper and unaltered part of the ilmenite. The characteristics of the sample nanotips vary greatly, though they were all extracted from a small surface area with dimensions of $5 \times 25 \mu \mathrm{m}$ on the same grain. The different distribution of space weathering products between the two nanotips is due to sampling different adjacent grain volumes, which were exposed to different degrees of space weathering even on this small scale. Both nanotips contain small nanophase Fe particles ( $\sim 3$ to $10 \mathrm{~nm}$ diameter), with these particles becoming less frequent with depth.

One of the nanotips contains a sequence of space weathering products, compositional zoning, and a void space ( $15 \mathrm{~nm}$ in diameter) which we interpret as a vesicle generated by solar wind irradiation. No noble gases were detected in this vesicle, although there is evidence for ${ }^{4} \mathrm{He}$ on the edge of this feature. The outermost layer of the grain is more enriched in the minor elements than the nanophase Fe area, suggesting that some of the rim material is sourced from outside of the ilmenite grain and is a true redeposition rim, formed by the condensation of vapor produced by the impact of micrometeorites on adjacent grains and by deposition of materials sputtered by energetic solar wind ions from the nearby grains.

In the other nanotip, $\mathrm{H}$-bearing ionic or molecular species such as $\mathrm{OH}^{+}$and $\mathrm{H}_{2} \mathrm{O}^{+}$, are present in the greatest amounts at a depth range of $40-50 \mathrm{~nm}$, fully consistent with $\mathrm{H}^{+}$solar wind implantation at typical energies (Fig. 1). The outermost layer of space-weathered soil comprised of O-rich minerals, such as that of this ilmenite grain, may be an important source of water on the Moon. 
This lunar soil grain exhibits the same space weathering features that have been well documented employing transmission electron microscope (TEM) studies of lunar and Itokawa asteroidal regolith grains. The zones we observe in the lunar material have the same compositional characteristics as material returned from Itokawa [8,9], but have different depth ranges. APT is an ideal analytical method to study nanoscale surface effects of precious samples, such as mission-returned samples like those presented herein and is a complementary technique to TEM.

\section{Acknowledgements}

The authors thank Ryan A. Zeigler (Johnson Space Center) for providing the lunar sample and Jean-Paul Benkert for preparing the ilmenite grain separate. We also thank Levke Kööp for maintaining the FIB/SEM. J.G. acknowledges support from the National Science Foundation Graduate Research Fellowship (DGE-1144082 and DGE-1746045). P.R.H. acknowledges the TAWANI Foundation who funded the Robert A. Pritzker Center through a major grant. Atom probe tomography was performed at the Northwestern University Center for Atom Probe Tomography (NUCAPT). The LEAP tomograph at NUCAPT was purchased and upgraded with grants from the NSF-MRI (DMR-0420532) and ONRDURIP (N00014-0400798, N00014-0610539, N00014-0910781, N00014-1712870) programs. NUCAPT received support from the MRSEC program (NSF DMR-1720139) at the Materials Research Center, the SHyNE Resource (NSF ECCS-2025633), and the Initiative for Sustainability and Energy (ISEN) at Northwestern University.

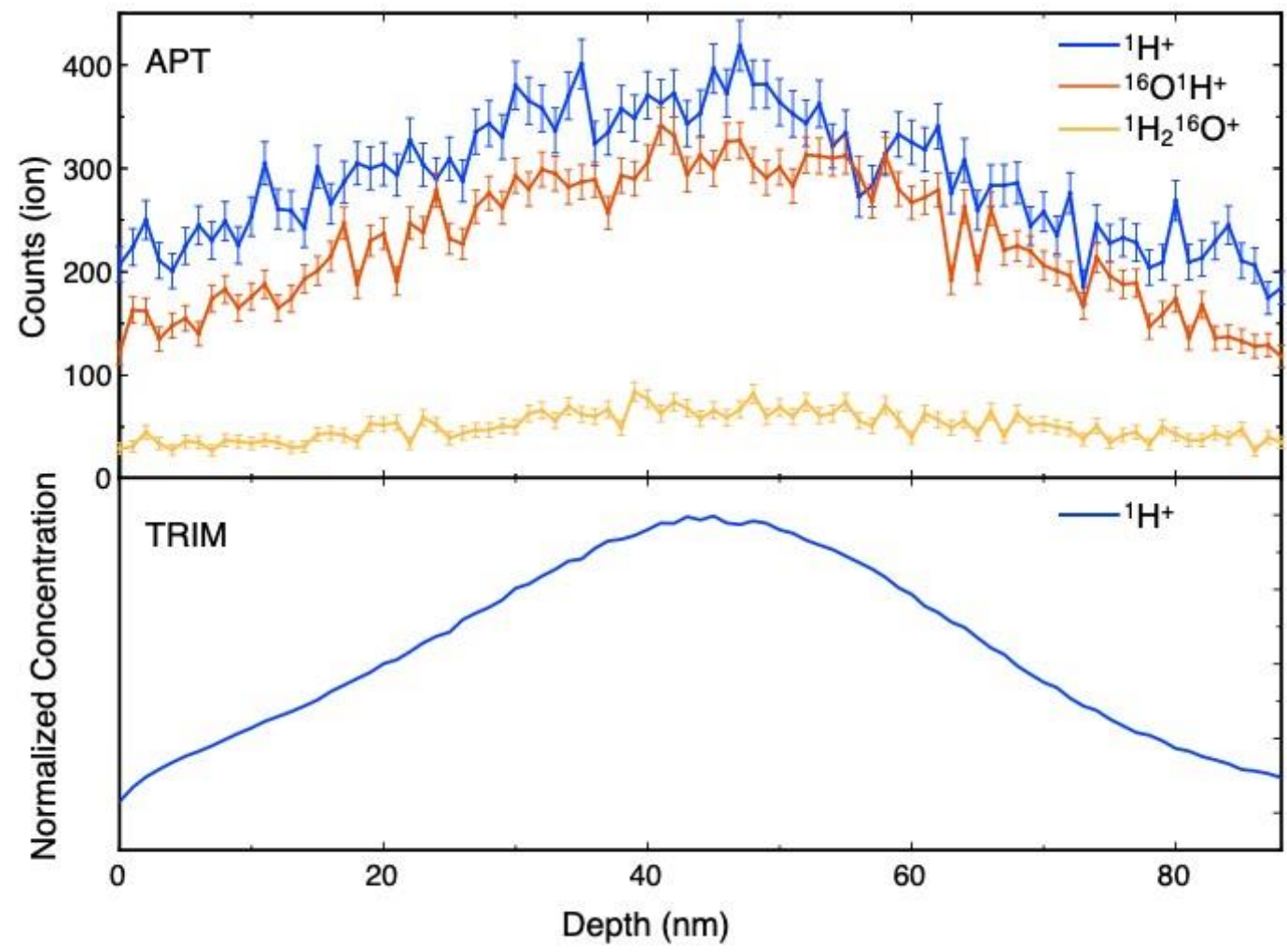

Figure 1. APT concentration profiles (top) of H-bearing ionic or molecular species along the axis normal to the grain's surface, with a shape consistent with simulated [7] H+ implantation at $4 \mathrm{keV}$ (bottom) into a material with properties comparable to ilmenite [5]. 


\section{References}

[1] Hapke, Bruce. "Space weathering from Mercury to the asteroid belt." Journal of Geophysical Research: Planets 106.E5 (2001): 10039-10073.

[2] Chapman, Clark R. "Space weathering of asteroid surfaces." Annual Review of Earth and Planetary Sciences 32 (2004).

[3] Bennett, Chris J., et al. "Space-weathering of solar system bodies: A laboratory perspective." Chemical reviews 113.12 (2013): 9086-9150.

[4] Pieters, Carle M., and Sarah K. Noble. "Space weathering on airless bodies." Journal of Geophysical Research: Planets 121.10 (2016): 1865-1884.

[5] Seidman, David N., and Krystyna Stiller. "An atom-probe tomography primer." Mrs Bulletin 34.10 (2009): 717-724.

[6] Greer, Jennika, et al. "Atom probe tomography of space-weathered lunar ilmenite grain surfaces." Meteoritics \& Planetary Science 55.2 (2020): 426-440.

[7] Ziegler James F., et al. SRIM: The stopping and range of ions in matter. (2012) Morrisville, North Carolina: Lulu Press Co. http://www.srim.org

[8] Noguchi, Takaaki, et al. "Incipient space weathering observed on the surface of Itokawa dust particles." Science 333.6046 (2011): 1121-1125.

[9] Noguchi, Takaaki, et al. "Space weathered rims found on the surfaces of the Itokawa dust particles." Meteoritics \& Planetary Science 49.2 (2014): 188-214. 